\title{
Fischöl im Mutterleib schützt vor Asthma
}

\section{Wenn Schwangere Fischöl einnehmen, verringern sie das Risiko des Kindes für Asthma bronchiale und rezidivierende obstruktive Bronchitiden.}

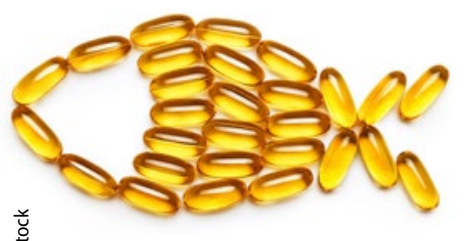

_ Eine geringe Zufuhr von langkettigen ungesättigten Fettsäuren (LCPUFA), wie sie in Fischöl vorkommen, gilt als Faktor für die zunehmende Prävalenz des Asthma brochiale. Um einen möglichen Einfluss der pränatalen Versorgung zu untersuchen, erhielten 736 schwangere Frauen in Dänemark randomisiert und doppelblind ab der 24. Schwangerschaftswoche täglich 2,4 g Fischöl oder Olivenöl. Nach der Geburt wurde die Verblindung für die Eltern aufgehoben.

Primäres Studienziel war der Einfluss auf die Entwicklung eines persistierenden Asthma bronchiale und/oder von rezidivierenden obstruktiven Bronchitiden. Die Kinder wurden dafür im Alter von 1 Woche, 1, 3, 6, 12, 18, 24, 30 und 36 Monaten und dann in jährlichen Abständen klinisch untersucht. Husten, Pfeifen und Atemnot wurden durch eine tägliche Registrierung über Fragebögen erfasst. Prick-Tests zur AllergieDiagnose wurden im Alter von 6 und 18 Monaten durchgeführt. Von 695 eingeschlossenen Kindern beendeten 95,5\% die dreijährige Beobachtungsphase.

Das Risiko, persistierende Atemwegsbronchitiden oder Asthma zu entwickeln, lag in der Verumgruppe bei 16,9\% und in der Kontrollgruppe bei 23,7\% - eine relative Risikoreduktion von 30,7\% ( $<<0,03)$. Der Effekt war am stärksten bei Müttern mit vergleichsweise niedrigen LCPUFA-Blutspiegeln zu Studienbeginn. Die Analyse der sekundären Zielparameter ergab eine signifikant geringere Prävalenz von Infektionen des unteren Atemwegstrakts in der Verumgruppe (31,7\% vs $39,1 \%$, $\mathrm{p}<0,03)$. Die Risiken für Asthmaexazerbationen, atopisches Ekzem oder Allergien wurden hingegen nicht beeinflusst.

- Bisgaard H, Stokholm J, Chawes BL et al. Fish oil-derived fatty acids in pregnacy and wheeze and asthma in offspring. N Engl J Med. 2016;375:2530-9

\section{KOMMENTAR}

Die Supplementierung von mehrfach ungesättigten Fettsäuren im letzten Trimester der Schwangerschaft reduziert das Risiko für rezidivierende obstruktive Bronchitiden, nicht-allergisches Asthma bronchiale und Infektionen des unteren Atemwegstrakts um fast ein Drittel. Die Befunde zeigen wieder einmal, dass pränatale Faktoren für eine spätere Krankheitsprägung verantwortlich sind. Es wäre allerdings noch interessant zu erfahren, ob bei Kindern von Müttern, die selbst an Asthma litten, ein höheres Risiko bestand.

Prof. em. Dr. med. Dr. h. c. D. Reinhardt

\section{Hier steht eine Anzeige.}

\author{
Springer
}

